\title{
Analysis of Cantilever Retaining wall using Matrix Displacement method and ANSYS
}

\author{
Shanmuga priya $\mathrm{R} \mathrm{K}^{1}$ \\ $\left\{\right.$ kna.civil@psgtech.ac.in $\left.{ }^{1}\right\}$ \\ Former PG Student (2015-17 batch), Department of Civil Engineering, PSG College of Technology \\ Coimbatore- 641004, India ', Professor, Department of Civil Engineering, PSG College of Technology \\ Coimbatore- 641004, India ${ }^{2}$
}

\begin{abstract}
Retaining walls are used for maintaining ground surface at different elevations on either side of it. The stability of earth retaining structures has become a serious problem due to scour, erosion etc., and the main reason for its instability is the improper analysis of such structures. Hence it is necessary to analyze the retaining walls in a proper manner by considering the various factors such as soil types, boundary conditions, size and type of retaining walls. Matrix displacement method and Finite Element method by ANSYS Software are used for the analysis of Cantilever retaining wall with uniform stem thickness and subjected to horizontal backfill. In the Matrix Displacement method, the cantilever retaining wall has been discretized into six numbers of four-noded isoparametric rectangular elements based on the aspect ratio and are analyzed as a plane strain problem. The element stiffness matrices for each element have been derived and are assembled to obtain the structural stiffness matrix of size 20x20 after applying the boundary conditions. From the structural stiffness matrix and the nodal loads, the nodal displacement, stresses and strains induced in each element are determined. In the finite element method, the cantilever retaining wall is modeled as a $2 \mathrm{D}$ plane strain model is meshed with quadrangle type of element at optimum edge size of $0.35 \mathrm{~m}$ is analyzed. The stresses induced in the toe and heel of the cantilever retaining wall are also determined by Static analysis and the stability of the retaining wall against overturning and sliding were checked. The stresses induced in the cantilever retaining wall obtained from the Matrix Displacement method, ANSYS and the Static analysis are compared.
\end{abstract}

Keywords: Matrix Displacement Method, Finite Element Method, plane strain problem, four noded isoparametric rectangular elements.

\section{Introduction}

Retaining walls are commonly used to retain soil on sloping sides, water in a pond or bulk products within a storage area. Stress induced in the retaining wall should not exceed the bearing capacity of the soil. Hence it is necessary to analyze the retaining walls. Retaining walls are classified as gravity, semi-gravity, cantilever and anchored. The Gravity wall is a wall that can resist the lateral earth force by its own weight. Semi-gravity walls are similar to gravity walls, except that they have some tension reinforcement which helps to reduce the wall thickness. Cantilever retaining walls rely on the structural components of the wall partially embedded in foundation to increase the passive resistance to resist lateral earth loads. 
Anchored walls are having the capacity to resist lateral earth loads by their structural components which are being restrained by tension elements connected to anchors and by partial embedment of their structural components into foundation material. For the present study, Cantilever retaining wall is analyzed analytically using Matrix displacement method and Finite Element Method by ANSYS software.

Anuj Chandiwala K, Gunvant solanki, Yash chaliawala [1] investigated Cantilever retaining walls are economically suited for heights up to $6 \mathrm{~m}$ whereas counterfort retaining walls are used for heights of $8 \mathrm{~m}-10 \mathrm{~m}$. The retaining wall with relieving platform is considered as the notable feature as it leads to discontinuous lateral earth press ure diagrams which in turn help in increasing the stability of the retaining wall.

Mustafa Ergun and Sevket Ates [4] investigated the stress analysis of shear wall using Matrix Displacement method.A shear wall is discretized into two Constant Strain Triangle elements. The Stiffness matrix and the load vector which are attained from external loads are calculated for each of the finite elements using Matrix Displacement Method. Finite element analysis of shear wall is done by ANSYS software. The result showed that this method is effective and preferable for the stress analysis of shell structures [12]..

\section{General Steps of the Finite Element Method}

The solution of a general continuum problem by the finite element method always follows the process given below. Initially, the structure is discretized into number of elements with suitable shape and size. As the displacement solution of a complex structure under any specified load conditions cannot be predicted exactly, some suitable solution within an element to approximate the unknown solution can be assumed. The stiffness matrix $[\mathrm{K}]$ and the load vector $\{\mathrm{F}\}$ of an element are derived and assembled in a suitable manner. After applying the boundary conditions, the nodal displacements can be derived from the relation, $[\mathrm{K}]\{\mathrm{u}\}=\{\mathrm{F}\}$. With the known nodal displacements, the element strains and stresses can be computed.

\section{Isoparametric Formulation of Plane Element Stiffness Matrix In Matrix Displacement Method}

The term isoparametric is derived from the use of the same shape functions to define the element shape and displacements within the element. Hence when the shape function is $\mathrm{u}=\mathrm{c}_{1}+\mathrm{c}_{2} \xi+\mathrm{c}_{3} \eta+\mathrm{c}_{4} \xi \eta$ for the displacement, $\quad \mathrm{x}=\mathrm{c}_{1}+\mathrm{c}_{2} \xi+\mathrm{c}_{3} \eta+\mathrm{c}_{4} \xi \eta$ is used for the description of a coordinate point in the plane element. The naturalcoordinate system $\xi-\eta$ is defined by element geometry. It is not defined by the element orientation in the global-coordinate system $x-y$. A basic square element in $\xi-\eta$ coordinates as shown in Fig. 1 is used as a parent element. Isoparametric formulation of the simple linear plane element stiffness matrix is discussed. 


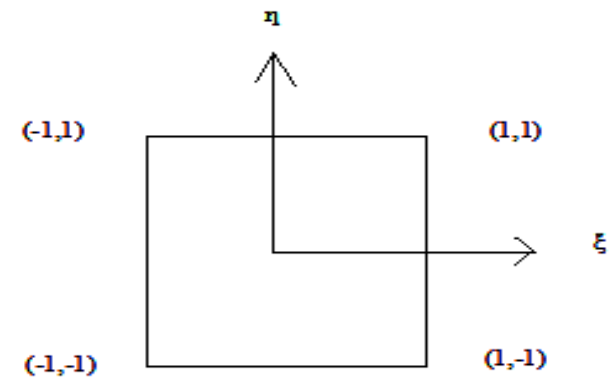

Fig. 1 Linear Square Element in $\xi-\eta$ Co-ordinates

\section{Step 1: Select Element Type}

First, the natural $\xi$ - $\eta$ coordinates are attached to the element, with the origin at the center of the element, as shown in Fig. 1. Consider the quadrilateral element as shown in Fig. 2 to have eight degrees of freedom, $\mathrm{u}_{1}, \mathrm{v}_{1}, \mathrm{u}_{2}, \mathrm{v}_{2}, \mathrm{u}_{3}, \mathrm{v}_{3}, \mathrm{u}_{4}$, and $\mathrm{v}_{4}$ associated with the global $\mathrm{x}$ and $\mathrm{y}$ directions as shown in Fig. 3 .

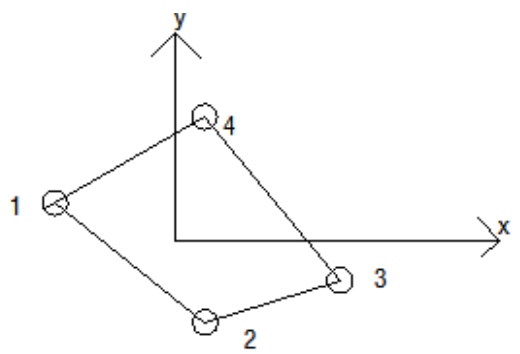

Fig. 2 Quadrilateral (Real) Element with Global Axis

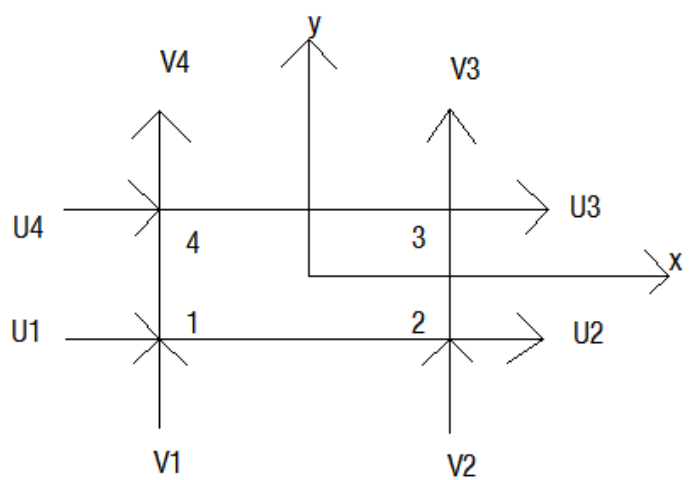

Fig. 3 Basic Four-Noded Rectangular Element with Nodal Degrees of Freedom 
The shape functions are used to map the square element shown in Fig. 1 in isoparametric coordinates $\xi$ and $\eta$ to the quadrilateral element shown in Fig. 2

For any quadrilateral,

$$
\begin{aligned}
& \mathrm{x}=\mathrm{c}_{1}+\mathrm{c}_{2} \xi+\mathrm{c}_{3} \eta+\mathrm{c}_{4} \xi \eta \\
& \mathrm{y}=\mathrm{c}_{5}+\mathrm{c}_{6} \xi+\mathrm{c}_{7} \eta+\mathrm{c}_{8} \xi \eta
\end{aligned}
$$

Solving for these constants, it is established as,

$$
\begin{aligned}
& x=N_{1} x_{1}+N_{2} x_{2}+N_{3} x_{3}+N_{4} x_{4} \\
& y=N_{1} y_{1}+N_{2} y_{2}+N_{3} y_{3}+N_{4} y_{4}
\end{aligned}
$$

From Fig.3,

$\mathrm{N}_{1}=\mathrm{k}$ (eqn. of line 3,4) (eqn. of line 2, 3)

$$
=\mathrm{k}(1-\eta)(1-\xi)
$$

At node 1, (-1,-1), $\mathrm{N}_{1}=1$

$1=\mathrm{k} * 2 * 2$

$\mathrm{k}=\frac{1}{4}$

$$
\begin{aligned}
& N_{1}=\frac{1}{4}(1-\eta)(1-\xi) \\
& N_{1}=\frac{1}{4}(1-\eta)(1-\xi)
\end{aligned}
$$

$\mathrm{N}_{2}=\mathrm{k}$ (eqn. of line 3, 4) (eqn. of line 1, 4)

$$
=\mathrm{k}(1-\eta)(1+\xi)
$$

At node 2, (1,-1), $\mathrm{N}_{2}=1$

$1=\mathrm{k} * 2 * 2$

$\mathrm{k}=\frac{1}{4}$

$$
N_{2}=\frac{1}{4}(1-\eta)(1+\xi)
$$


$\mathrm{N}_{3}=\mathrm{k}$ (eqn. of line 1, 2) (eqn. of line 1, 4)

$$
=\mathrm{k}(1+\eta)(1+\xi)
$$

At node 3, (1,1), $\mathrm{N}_{3}=1$

$1=\mathrm{k} * 2 * 2$

$\mathrm{k}=\frac{1}{4}$

$$
N_{3}=\frac{1}{4}(1+\eta)(1+\xi)
$$

$\mathrm{N}_{4}=\mathrm{k}$ (eqn. of line 1, 2) (eqn. of line 2, 3)

$$
=\mathrm{k}(1+\eta)(1-\xi)
$$

At node $4,(-1,1), \mathrm{N}_{4}=1$

$$
1=\mathrm{k} * 2 * 2
$$

$\mathrm{k}=\frac{1}{4}$

$$
N_{4}=\frac{1}{4}(1+\eta)(1-\xi)
$$

$\mathrm{N}_{1}+\mathrm{N}_{2}+\mathrm{N}_{3}+\mathrm{N}_{4}=1$ for all values of $\xi$ and $\eta$. The shape functions $\mathrm{N}_{1}$ through $\mathrm{N}_{4}$ have the properties that $\mathrm{Ni}=1$ at node $\mathrm{i}$ and equal to zero at all other nodes.

\section{Step 2: Select Displacement Functions}

The displacement functions within an element are defined by the same shape functions which are used to define the element shape.

$$
\left\{\begin{array}{l}
u \\
v
\end{array}\right\}=\left[\begin{array}{cccccccc}
N_{1} & 0 & N_{2} & 0 & N_{3} & 0 & N_{4} & 0 \\
0 & N_{1} & 0 & N_{2} & 0 & N_{3} & 0 & N_{4}
\end{array}\right]\left\{\begin{array}{l}
u_{1} \\
v_{1} \\
u_{2} \\
v_{2} \\
u_{3} \\
v_{3} \\
u_{4} \\
v_{4}
\end{array}\right\}
$$

where $\mathrm{u}$ and $\mathrm{v}$ are displacements parallel to the global $\mathrm{x}$ and $\mathrm{y}$ coordinates. The displacement of an interior point located at $x, y$ in the element shown in Fig. 3 is described by $u$ and $v$. Nodal displacements are denoted by $\mathrm{u}_{1}, \mathrm{v}_{1}, \mathrm{u}_{2}, \mathrm{v}_{2}, \mathrm{u}_{3}, \mathrm{v}_{3}, \mathrm{u}_{4}$ and $\mathrm{v}_{4}$ respectively. 


\section{Step 3: Characterize the Strain-Displacement and Stress-Strain Relationships}

Strain-Displacement matrix, $[\mathrm{B}]$ has to be formulated to evaluate the stiffness matrix, K. Since, it becomes tedious to write the shape functions in terms of the $x$ and $y$ coordinates, the formulation will be carried out in terms of the isoparametric coordinates $\xi$ and $\eta$. To construct an element stiffness matrix, the strains must be defined in terms of the derivatives of the displacements with respect to the $\mathrm{x}$ and $\mathrm{y}$ coordinates. The displacements are now functions of the $\xi$ and $\eta$ coordinates given by equations 1 - 4. Hence, $\partial \phi / \partial \mathrm{x}$ and $\partial \phi / \partial \mathrm{y}$ have to be determined, where, in general, $\phi$ is a function representing the displacement functions $\mathrm{u}$ or $\mathrm{v}$. However, $\mathrm{u}$ and $\mathrm{v}$ are now expressed in terms of $\xi$ and $\eta$. Therefore, the chain rule of differentiation is applied to express $\xi$ and $\eta$ as functions of $\mathrm{x}$ and $\mathrm{y}$.

$$
\begin{aligned}
& \frac{\partial \phi}{\partial \xi}=\frac{\partial \phi}{\partial x} * \frac{\partial x}{\partial \xi}+\frac{\partial \phi}{\partial y} * \frac{\partial y}{\partial \xi} \\
& \frac{\partial \phi}{\partial \eta}=\frac{\partial \phi}{\partial x} * \frac{\partial x}{\partial \eta}+\frac{\partial \phi}{\partial y} * \frac{\partial y}{\partial \eta} \\
& \left\{\begin{array}{l}
\frac{\partial \phi}{\partial \xi} \\
\frac{\partial \phi}{\partial \eta}
\end{array}\right\}=\left[\begin{array}{ll}
\frac{\partial x}{\partial \xi} & \frac{\partial y}{\partial \xi} \\
\frac{\partial x}{\partial \eta} & \frac{\partial y}{\partial \eta}
\end{array}\right]\left\{\begin{array}{l}
\frac{\partial \phi}{\partial x} \\
\frac{\partial \phi}{\partial y}
\end{array}\right\} \\
& \mathrm{x}=\mathrm{N}_{1} \mathrm{x}_{1}+\mathrm{N}_{2} \mathrm{x}_{2}+\mathrm{N}_{3} \mathrm{x}_{3}+\mathrm{N}_{4} \mathrm{x}_{4} \\
& \frac{\partial x}{\partial \xi}=\frac{\partial N_{1}}{\partial \xi} x_{1}+\frac{\partial N_{2}}{\partial \xi} x_{2}+\frac{\partial N_{3}}{\partial \xi} x_{3}+\frac{\partial N_{4}}{\partial \xi} x_{4} \\
& \frac{\partial x}{\partial \xi}=\frac{1}{4}\left(-(1-\eta) x_{1}+(1-\eta) x_{2}+(1+\eta) x_{3}-(1+\eta) x_{4}\right)
\end{aligned}
$$

Similarly,

$$
\begin{aligned}
& \frac{\partial x}{\partial \eta}=\frac{1}{4}\left(-(1-\xi) x_{1}-(1-\xi) x_{2}+(1+\xi) x_{3}+(1-\xi) x_{4}\right) \\
& \frac{\partial y}{\partial \xi}=\frac{\partial N_{1}}{\partial \xi} y_{1}+\frac{\partial N_{2}}{\partial \xi} y_{2}+\frac{\partial N_{3}}{\partial \xi} y_{3}+\frac{\partial N_{4}}{\partial \xi} y_{4} \\
& \frac{\partial y}{\partial \xi}=\frac{1}{4}\left(-(1-\eta) y_{1}+(1-\eta) y_{2}+(1+\eta) y_{3}-(1+\eta) y_{4}\right) \\
& \frac{\partial y}{\partial \eta}=\frac{1}{4}\left(-(1-\xi) y_{1}-(1+\xi) y_{2}+(1+\xi) y_{3}+(1-\xi) y_{4}\right)
\end{aligned}
$$




$$
\left\{\begin{array}{l}
\frac{\partial \varphi}{\partial x} \\
\frac{\partial \varphi}{\partial y}
\end{array}\right\}=[J]^{-1}\left\{\begin{array}{l}
\frac{\partial \varphi}{\partial \xi} \\
\frac{\partial \varphi}{\partial \eta}
\end{array}\right\}
$$

Since $\phi$ is a function representing the displacement functions $\mathrm{u}$ or $\mathrm{v}$,

$$
\begin{aligned}
& \left\{\begin{array}{l}
\frac{\partial u}{\partial x} \\
\frac{\partial u}{\partial y}
\end{array}\right\}=[J]^{-1}\left\{\begin{array}{l}
\frac{\partial u}{\partial \xi} \\
\frac{\partial u}{\partial \eta}
\end{array}\right\},\left\{\begin{array}{l}
\frac{\partial v}{\partial x} \\
\frac{\partial v}{\partial y}
\end{array}\right\}=[J]^{-1}\left\{\begin{array}{c}
\frac{\partial v}{\partial \xi} \\
\frac{\partial v}{\partial \eta}
\end{array}\right\} \\
& \varepsilon_{x}=\frac{\partial u}{\partial x} \quad \varepsilon_{y}=\frac{\partial v}{\partial y}, \quad \gamma_{x y}=\frac{\partial v}{\partial x}+\frac{\partial u}{\partial y}
\end{aligned}
$$

$$
\begin{gathered}
\{\varepsilon\}=\left\{\begin{array}{l}
\varepsilon_{x} \\
\varepsilon_{y} \\
\gamma_{x y}
\end{array}\right\} \\
\left.\left\{\begin{array}{l}
\frac{\partial u}{\partial x} \\
\frac{\partial u}{\partial y} \\
\frac{\partial v}{\partial x} \\
\frac{\partial v}{\partial y}
\end{array}\right\}=\left[\begin{array}{cc}
J^{-1} & 0 \\
0 & J^{-1}
\end{array}\right]\left\{\begin{array}{l}
\frac{\partial u}{\partial \xi} \\
\frac{\partial u}{\partial \eta} \\
\frac{\partial v}{\partial \xi} \\
\frac{\partial v}{\partial \xi}
\end{array}\right\},\{\varepsilon\}=\left[\begin{array}{llll}
1 & 0 & 0 & 0 \\
0 & 0 & 0 & 1 \\
0 & 1 & 1 & 0
\end{array}\right], \begin{array}{l}
\frac{\partial u}{\partial x} \\
\frac{\partial u}{\partial y} \\
\frac{\partial v}{\partial x} \\
\frac{\partial v}{\partial y}
\end{array}\right\}
\end{gathered}
$$

$$
=\left[\begin{array}{llll}
1 & 0 & 0 & 0 \\
0 & 0 & 0 & 1 \\
0 & 1 & 1 & 0
\end{array}\right] \frac{1}{|J|}\left[\begin{array}{cccc}
J_{22} & -J_{12} & 0 & 0 \\
-J_{21} & J_{11} & 0 & 0 \\
0 & 0 & J_{22} & -J_{12} \\
0 & 0 & -J_{21} & J_{11}
\end{array}\right]\left\{\begin{array}{l}
\frac{\partial u}{\partial \xi} \\
\frac{\partial u}{\partial \eta} \\
\frac{\partial v}{\partial \xi} \\
\frac{\partial v}{\partial \xi}
\end{array}\right\}
$$




$$
\begin{aligned}
& \left\{\begin{array}{l}
\frac{\partial u}{\partial \xi} \\
\frac{\partial u}{\partial \eta} \\
\frac{\partial v}{\partial \xi} \\
\frac{\partial v}{\partial \xi}
\end{array}\right\}=\left[\begin{array}{cccccccc}
\frac{\partial N_{1}}{\partial \xi} & 0 & \frac{\partial N_{2}}{\partial \xi} & 0 & \frac{\partial N_{3}}{\partial \xi} & 0 & \frac{\partial N_{4}}{\partial \xi} & 0 \\
\frac{\partial N_{1}}{\partial \eta} & 0 & \frac{\partial N_{2}}{\partial \eta} & 0 & \frac{\partial N_{3}}{\partial \eta} & 0 & \frac{\partial N_{4}}{\partial \eta} & 0 \\
0 & \frac{\partial N_{1}}{\partial \xi} & 0 & \frac{\partial N_{2}}{\partial \xi} & 0 & \frac{\partial N_{3}}{\partial \xi} & 0 & \frac{\partial N_{4}}{\partial \xi} \\
0 & \frac{\partial N_{1}}{\partial \eta} & 0 & \frac{\partial N_{2}}{\partial \eta} & 0 & \frac{\partial N_{3}}{\partial \eta} & 0 & \frac{\partial N_{4}}{\partial \eta}
\end{array}\right]\left\{\begin{array}{l}
u_{1} \\
v_{1} \\
u_{2} \\
y_{2} \\
u_{3} \\
v_{3} \\
u_{4} \\
v_{4}
\end{array}\right\} \\
& {[A]=\left[\begin{array}{llll}
1 & 0 & 0 & 0 \\
0 & 0 & 0 & 1 \\
0 & 1 & 1 & 0
\end{array}\right] \frac{1}{|J|}\left[\begin{array}{cccc}
J_{22} & -J_{12} & 0 & 0 \\
-J_{21} & J_{11} & 0 & 0 \\
0 & 0 & J_{22} & -J_{12} \\
0 & 0 & -J_{21} & J_{11}
\end{array}\right]} \\
& {[G]=\left[\begin{array}{cccccccc}
\frac{\partial N_{1}}{\partial \xi} & 0 & \frac{\partial N_{2}}{\partial \xi} & 0 & \frac{\partial N_{3}}{\partial \xi} & 0 & \frac{\partial N_{4}}{\partial \xi} & 0 \\
\frac{\partial N_{1}}{\partial \eta} & 0 & \frac{\partial N_{2}}{\partial \eta} & 0 & \frac{\partial N_{3}}{\partial \eta} & 0 & \frac{\partial N_{4}}{\partial \eta} & 0 \\
0 & \frac{\partial N_{1}}{\partial \xi} & 0 & \frac{\partial N_{2}}{\partial \xi} & 0 & \frac{\partial N_{3}}{\partial \xi} & 0 & \frac{\partial N_{4}}{\partial \xi} \\
0 & \frac{\partial N_{1}}{\partial \eta} & 0 & \frac{\partial N_{2}}{\partial \eta} & 0 & \frac{\partial N_{3}}{\partial \eta} & 0 & \frac{\partial N_{4}}{\partial \eta}
\end{array}\right]} \\
& \text { In actual, }\{\varepsilon\}=[B]\{\mathrm{q}\} \\
& \text { Here, }\{\varepsilon\}=[A][G]\{q\} \\
& \text { Therefore, }[\mathrm{B}]=[\mathrm{A}][\mathrm{G}]
\end{aligned}
$$

For plane strain problem, since, dimension in z-direction is very large,

$$
\varepsilon_{x}=\gamma_{z x}=\gamma_{z y}=0
$$

$$
\begin{aligned}
& \varepsilon_{x}=\frac{1}{E}\left(\sigma_{x x}-v \sigma_{y y}-v \sigma_{z z}\right) \\
& \varepsilon_{y}=\frac{1}{E}\left(\sigma_{y y}-v \sigma_{x x}-v \sigma_{z z}\right) \\
& \varepsilon_{z}=\frac{1}{E}\left(\sigma_{z z}-v \sigma_{y y}-v \sigma_{x x}\right)
\end{aligned}
$$

From Eqn. (5), $\sigma_{z z}=v\left(\sigma_{x x}+\sigma_{y y}\right)$

$$
\begin{aligned}
& \varepsilon_{x}=\frac{1}{E}\left(\left(1-v^{2}\right) \sigma_{x x}-v(1+v) \sigma_{y y}\right) \\
& \varepsilon_{y}=\frac{1}{E}\left(\left(1-v^{2}\right) \sigma_{y y}-v(1+v) \sigma_{x x}\right)
\end{aligned}
$$




$$
\begin{aligned}
& \tau_{x y}=G \gamma_{x y} \\
& \tau_{x y}=\frac{E}{2(1+v)} \gamma_{x y} \\
& \left\{\begin{array}{c}
\varepsilon_{x} \\
\varepsilon_{y}
\end{array}\right\}=\frac{1+v}{E}\left[\begin{array}{cc}
1-v & -v \\
-v & 1-v
\end{array}\right]\left\{\begin{array}{l}
\sigma_{x x} \\
\sigma_{y y}
\end{array}\right\} \\
& \left\{\begin{array}{l}
\sigma_{x x} \\
\sigma_{y y} \\
\tau_{x y}
\end{array}\right\}=\frac{E}{(1+v)(1-2 v)}\left[\begin{array}{ccc}
1-v & v & 0 \\
v & 1-v & 0 \\
0 & 0 & \frac{1-2 v}{2}
\end{array}\right]\left\{\begin{array}{l}
\varepsilon_{x} \\
\varepsilon_{y} \\
\gamma_{x y}
\end{array}\right\} \\
& \text { Constitutive Matrix },[\mathrm{C}]=\frac{E}{(1+v)(1-2 v)}\left[\begin{array}{ccc}
1-v & v & 0 \\
v & 1-v & 0 \\
0 & 0 & \frac{1-2 v}{2}
\end{array}\right] \\
& {[J]=\left[\begin{array}{ll}
\frac{\partial x}{\partial \xi} & \frac{\partial y}{\partial \xi} \\
\frac{\partial x}{\partial \eta} & \frac{\partial y}{\partial \eta}
\end{array}\right]}
\end{aligned}
$$

\section{Step 4: Derive the Element Stiffness Matrix}

For an element with a constant thickness t, the stiffness matrix in terms of $\xi, \eta$

$$
\text { coordinates will be }[\mathrm{K}]=\int_{-1}^{1} \int_{-1}^{1}[\mathrm{~B}][\mathrm{C}][\mathrm{B}] \mathrm{t}|\mathrm{J}| \mathrm{d} \xi \mathrm{d} \eta
$$

\section{Analysis Of Cantilever Retaining Wall}

Cantilever retaining walls up to $6 \mathrm{~m}$ in height are usually built as shown in Fig.4. These consist of concrete or masonry wall of uniform thickness. Cantilever Retaining wall is discretized into six numbers of four - noded isoparametric rectangular elements. 


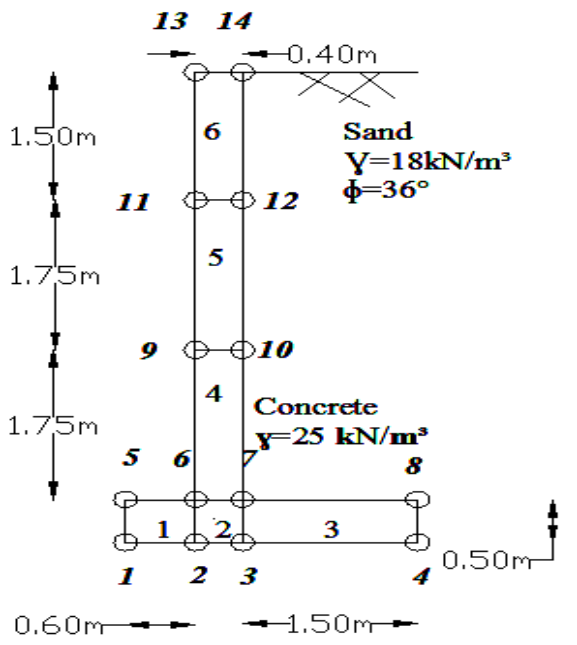

Fig. 4 Cantilever Retaining Wall

A cantilever retaining wall of height $5.5 \mathrm{~m}$, thickness $0.4 \mathrm{~m}$, toe width $0.6 \mathrm{~m}$, heel width $1.5 \mathrm{~m}$ is considered [5]. It is backfilled with sand that has total unit weight of 25 $\mathrm{kN} / \mathrm{m}^{3}$ and the characteristic compressive strength of $25 \mathrm{~N} / \mathrm{mm}^{2}$. The Poisson's ratio of the concrete is 0.2 and its Modulus of elasticity is $25 \times 10^{6} \mathrm{kN} / \mathrm{m}^{2}$.

\section{A. Formulation of Structural Stiffness Matrix of Cantilever Retaining Wall}

\section{Element1:}

The Element 1 in the Cantilever retaining wall with its global coordinates is shown in Fig. 5 and is considered as a Real Element 1.

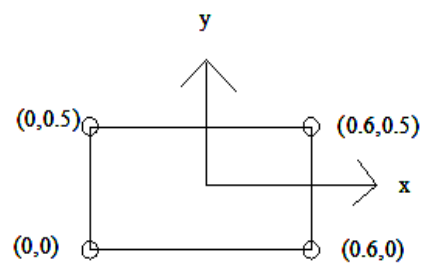

Fig. 5 Real Element 1 
$\left\{\begin{array}{l}\mathrm{u} \\ \mathrm{v}\end{array}\right\}=\left[\begin{array}{cccccccc}\mathrm{N}_{1} & 0 & \mathrm{~N}_{2} & 0 & \mathrm{~N}_{3} & 0 & \mathrm{~N}_{4} & 0 \\ 0 & \mathrm{~N}_{1} & 0 & \mathrm{~N}_{2} & 0 & \mathrm{~N}_{3} & 0 & \mathrm{~N}_{4}\end{array}\right]\left\{\begin{array}{l}\mathrm{u}_{1} \\ \mathrm{v}_{1} \\ \mathrm{u}_{2} \\ \mathrm{v}_{2} \\ \mathrm{u}_{3} \\ \mathrm{v}_{3} \\ \mathrm{u}_{4} \\ \mathrm{v}_{4}\end{array}\right\}$

$\left\{\begin{array}{l}x_{1} \\ x_{2} \\ x_{3} \\ x_{4}\end{array}\right\}=\left\{\begin{array}{c}0 \\ 0.6 \\ 0.6 \\ 0\end{array}\right\} m \quad\left\{\begin{array}{l}y_{1} \\ y_{2} \\ y_{3} \\ y_{4}\end{array}\right\}=\left\{\begin{array}{c}0 \\ 0 \\ 0.5 \\ 0.5\end{array}\right\} m$

Jacobian matrix, $[\mathrm{J}]=\left[\begin{array}{ll}\frac{\partial \mathrm{x}}{\partial \xi} & \frac{\partial \mathrm{y}}{\partial \xi} \\ \frac{\partial \mathrm{x}}{\partial \eta} & \frac{\partial \mathrm{y}}{\partial \eta}\end{array}\right]$

Substituting the values for co-ordinates,

Jacobian matrix, $[\mathrm{J}]=\left[\begin{array}{cc}0.3 & 0 \\ 0 & 0.25\end{array}\right]$

$|\mathrm{J}|=0.075$

$[A]=\left[\begin{array}{llll}1 & 0 & 0 & 0 \\ 0 & 0 & 0 & 1 \\ 0 & 1 & 1 & 0\end{array}\right] \frac{1}{|J|}\left[\begin{array}{cccc}\mathrm{J}_{22} & -\mathrm{J}_{12} & 0 & 0 \\ -\mathrm{J}_{21} & \mathrm{~J}_{11} & 0 & 0 \\ 0 & 0 & \mathrm{~J}_{22} & -\mathrm{J}_{12} \\ 0 & 0 & -\mathrm{J}_{21} & \mathrm{~J}_{11}\end{array}\right]$

$[\mathrm{A}]=\left[\begin{array}{cccc}3.333 & 0 & 0 & 0 \\ 0 & 0 & 0 & 4 \\ 0 & 4 & 3.333 & 0\end{array}\right]$

$[\mathrm{G}]=\left(\frac{1}{4}\right)\left[\begin{array}{cccccccc}-(1-\eta) & 0 & (1-\eta) & 0 & (1+\eta) & 0 & -(1+\eta) & 0 \\ -(1-\xi) & 0 & -(1+\xi) & 0 & (1+\xi) & 0 & (1-\xi) & 0 \\ 0 & -(1-\eta) & 0 & (1-\eta) & 0 & (1+\eta) & 0 & -(1+\eta) \\ 0 & -(1-\xi) & 0 & -(1+\xi) & 0 & (1+\xi) & 0 & (1-\xi)\end{array}\right]$

$[\mathrm{B}]=[\mathrm{A}][\mathrm{G}]$

Constitutive matrix for the Cantilever retaining wall with uniform stem thickness,

$[C]=34.722 \times 10^{6}\left[\begin{array}{ccc}0.8 & 0.2 & 0 \\ 0.2 & 0.8 & 0 \\ 0 & 0 & 0.3\end{array}\right] \mathrm{kN} / \mathrm{m}^{2}$

The Stiffness matrix for the element 1 for unit thickness is determined as

$[\mathrm{K}]=\int_{-1}^{1} \int_{-1}^{1}[\mathrm{~B}]^{\mathrm{T}}[\mathrm{C}][\mathrm{B}] \mathrm{t}|\mathrm{J}| \mathrm{d} \xi \mathrm{d} \eta$ 


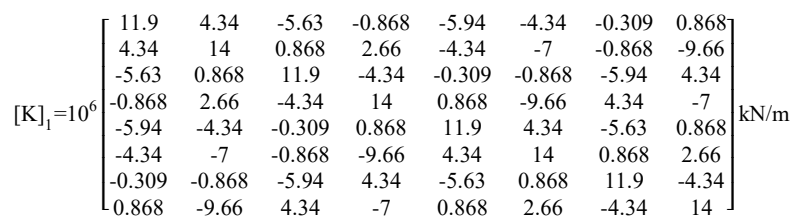

Similarly, the stiffness matrix for all other elements can also be determined as
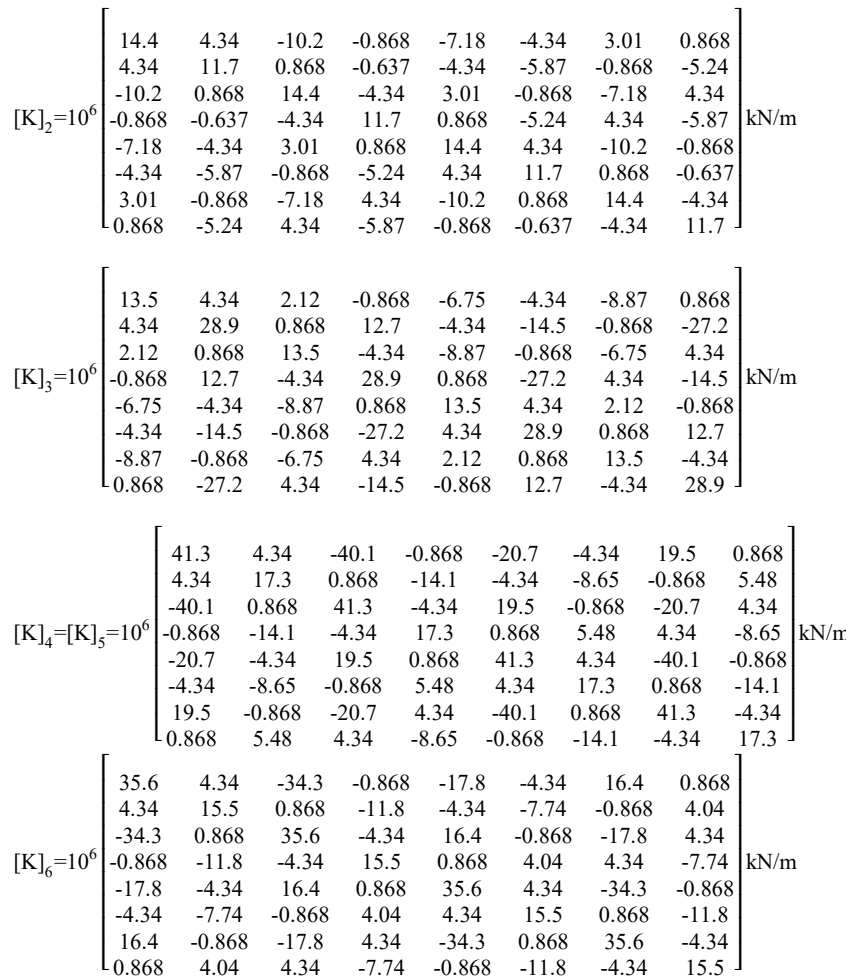

The stiffness matrix for the entire structure is obtained by assembling the element stiffness matrix of 6 elements by superposition method. The size of structural stiffness matrix is $28 \times 28$ as the size of each element stiffness matrix is $8 \times 8$. After applying the boundary conditions, it is reduced to $20 \mathrm{x} 20$.The final stiffness matrix will be

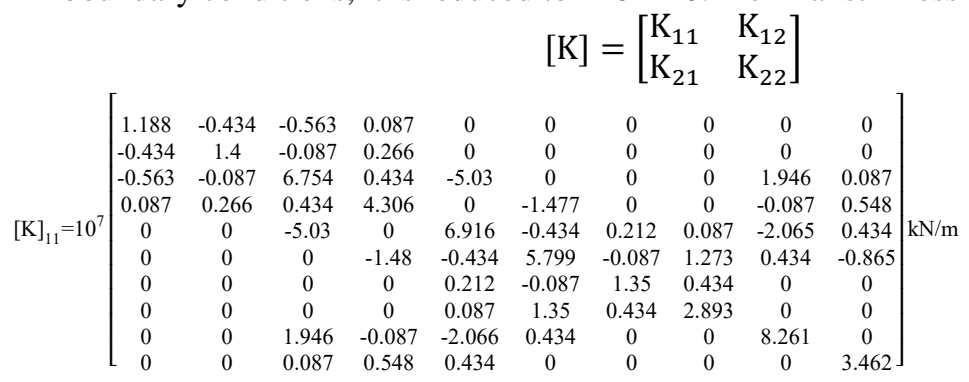




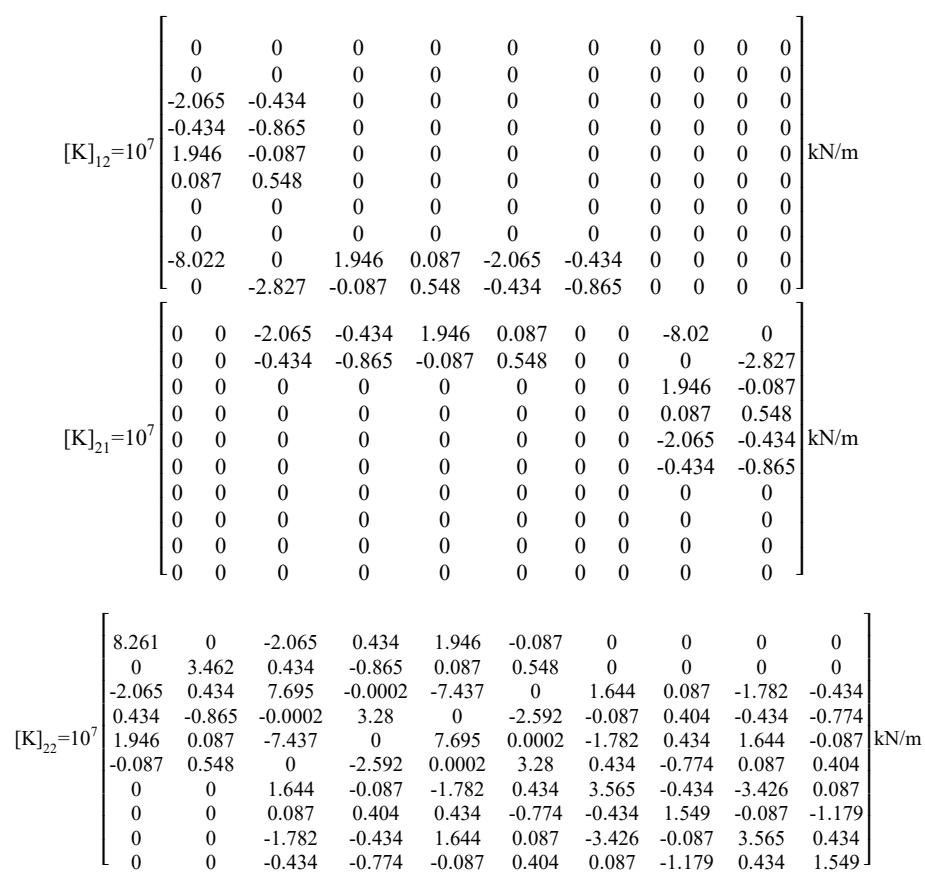

\section{A. Calculation of Nodal Forces and Nodal Displacements [5,6]}

Cantilever retaining wall with uniform stem thickness which is retaining sand as backfill and is subjected to an active earth pressure is shown in Fig.6

Active earth pressure coefficient, $\mathrm{k}_{\mathrm{a}}=\frac{1-\sin \phi}{1+\sin \phi}=0.26$

Active earth pressure, $\mathrm{p}_{\mathrm{a}}=\mathrm{k}_{\mathrm{a}} \gamma \mathrm{h}=0.26 \times 18 \times 5.5=25.74 \mathrm{kN} / \mathrm{m}^{2}$
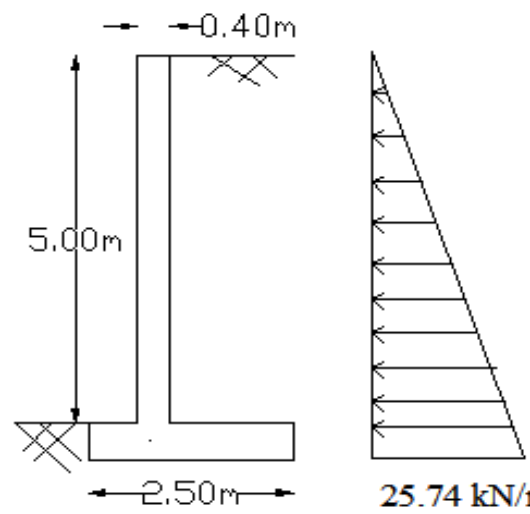

$25.74 \mathrm{kN} / \mathrm{m}^{2}$

Fig. 6 Cantilever Retaining Wall with Uniform Stem Thickness subjected to an Active Earth Pressure In general, $\{\mathrm{Q}\}=\int[\mathrm{N}]^{\mathrm{T}}\{\mathrm{T}\} \mathrm{ds}$ 


$$
\begin{aligned}
& \left\{\mathrm{Q}_{\mathrm{x}}\right\}=\int_{-\mathrm{b}}^{\mathrm{b}}[\mathrm{N}]^{\mathrm{T}}\left\{\mathrm{p}_{\mathrm{x}}\right\} \mathrm{dy} \\
& \left\{\begin{array}{l}
Q_{14 x} \\
Q_{12 x} \\
Q_{10 x} \\
Q_{7 x}
\end{array}\right\}=b \int_{-1}^{1}\left\{\begin{array}{c}
\left(\frac{9}{16}\right) *\left(\eta-\left(\frac{1}{3}\right)\right) *\left(\eta+\left(\frac{1}{3}\right)\right) *(1+\eta) \\
\left(\frac{27}{16}\right) *(1-\eta) *(1+\eta) *\left(\eta+\left(\frac{1}{3}\right)\right) \\
\left(\frac{9}{16}\right) *\left(\eta-\left(\frac{1}{3}\right)\right) *\left(\eta+\left(\frac{1}{3}\right)\right) *(1-\eta)
\end{array}\right\} \\
& \langle(1+\eta) / 2 \mid(1-\eta) / 2\rangle\left\{\begin{array}{c}
0 \\
-23.4
\end{array}\right\} d \eta \\
& =-23.4 *\left(\frac{5}{2}\right)\left\{\begin{array}{c}
\frac{1}{30} \\
\frac{3}{20} \\
\frac{3}{5} \\
\frac{13}{60}
\end{array}\right\} \\
& \left\{\begin{array}{l}
Q_{14 \times} \\
Q_{12 \times} \\
Q_{10 x} \\
Q_{7 x}
\end{array}\right\}=\left\{\begin{array}{c}
-0.78 \\
-3.51 \\
-14.04 \\
-5.07
\end{array}\right\} \mathbf{k N}
\end{aligned}
$$

\section{Loads Due to Self Weight:}

Point load is acting at the centroid of an element 1 (at $\xi=\eta=0$, since it is a perfect rectangular element).

$\{\mathrm{Q}\}=[\mathrm{N}]^{\mathrm{T}}\{\mathrm{F}\}$

$=25 * 0.6 * 0.5 * 1=7.5 \mathrm{kN}$

$=\left[\begin{array}{cccccccc}\mathrm{N}_{1} & \mathrm{~N}_{2} & \mathrm{~N}_{3} & \mathrm{~N}_{4} & 0 & 0 & 0 & 0 \\ 0 & 0 & 0 & 0 & \mathrm{~N}_{1} & \mathrm{~N}_{2} & \mathrm{~N}_{3} & \mathrm{~N}_{4}\end{array}\right]^{\mathrm{T}}\left\{\begin{array}{c}0 \\ -7.5\end{array}\right\}$ 
$=\left(\frac{1}{4}\right)\left[\begin{array}{llllllll}1 & 1 & 1 & 1 & 0 & 0 & 0 & 0 \\ 0 & 0 & 0 & 0 & 1 & 1 & 1 & 1\end{array}\right]^{\mathrm{T}}\left\{\begin{array}{c}0 \\ -7.5\end{array}\right\}$

$\left\{\begin{array}{l}\mathrm{Q}_{1 \mathrm{x}} \\ \mathrm{Q}_{2 \mathrm{x}} \\ \mathrm{Q}_{6 \mathrm{x}} \\ \mathrm{Q}_{5 \mathrm{x}} \\ \mathrm{Q}_{1 \mathrm{y}} \\ \mathrm{Q}_{2 \mathrm{y}} \\ \mathrm{Q}_{6 \mathrm{y}} \\ \mathrm{Q}_{5 \mathrm{y}}\end{array}\right\}=\left\{\begin{array}{c}0 \\ 0 \\ 0 \\ 0 \\ -1.875 \\ -1.875 \\ -1.875 \\ -1.875\end{array}\right\} \mathrm{kN}$

Similarly, for all other elements, nodal loads due to self weight can be determined.

$\left\{\begin{array}{l}Q_{2 y} \\ Q_{3 y} \\ Q_{4 y} \\ Q_{6 y}\end{array}\right\}=\left\{\begin{array}{l}-1.25 \\ -1.25 \\ -1.25 \\ -1.25\end{array}\right\} \mathrm{kN} ;\left\{\begin{array}{l}Q_{3 y} \\ Q_{4 y} \\ Q_{8 y} \\ Q_{7 y}\end{array}\right\}=\left\{\begin{array}{l}-4.688 \\ -4.688 \\ -4.688 \\ -4.688\end{array}\right\} \mathrm{kN} ;$

$\left\{\begin{array}{c}Q_{6 y} \\ Q_{7 y} \\ Q_{10 y} \\ Q_{9 y}\end{array}\right\}=\left\{\begin{array}{l}-4.375 \\ -4.375 \\ -4.375 \\ -4.375\end{array}\right\} \mathrm{kN}$

$\left\{\begin{array}{l}Q_{9 y} \\ Q_{10 y} \\ Q_{12 y} \\ Q_{11 y}\end{array}\right\}=\left\{\begin{array}{l}-4.375 \\ -4.375 \\ -4.375 \\ -4.375\end{array}\right\} \mathrm{kN} ;\left\{\begin{array}{l}Q_{11 y} \\ Q_{12 y} \\ Q_{14 y} \\ Q_{13 y}\end{array}\right\}=\left\{\begin{array}{l}-3.75 \\ -3.75 \\ -3.75 \\ -3.75\end{array}\right\} \mathrm{kN}$

Finally,

$\left\{\begin{array}{l}Q_{1 x} \\ Q_{1 y} \\ Q_{2 x} \\ Q_{2 y} \\ Q_{3 x} \\ Q_{3 y} \\ Q_{4 x} \\ Q_{4 y} \\ Q_{5 x} \\ Q_{5 y}\end{array}\right\}=\left\{\begin{array}{c}0 \\ -1.875 \\ 0 \\ -3.125 \\ 0 \\ -5.938 \\ -6.24 \\ -4.688 \\ -5.07 \\ -1.875\end{array}\right\} \mathrm{kN}$ 


$$
\left\{\begin{array}{l}
\mathrm{Q}_{6 \mathrm{x}} \\
\mathrm{Q}_{6 \mathrm{y}} \\
\mathrm{Q}_{7 \mathrm{x}} \\
\mathrm{Q}_{7 \mathrm{y}} \\
\mathrm{Q}_{8 \mathrm{x}} \\
\mathrm{Q}_{8 \mathrm{y}} \\
\mathrm{Q}_{9 \mathrm{x}} \\
\mathrm{Q}_{9 \mathrm{y}} \\
\mathrm{Q}_{10 \mathrm{x}} \\
\mathrm{Q}_{10 \mathrm{y}}
\end{array}\right\}=\left\{\begin{array}{c}
0 \\
-7.5 \\
0 \\
-77.813 \\
-6.045 \\
-72.188 \\
0 \\
-8.75 \\
-14.04 \\
-8.75
\end{array}\right\} \mathrm{kN} ;\left\{\begin{array}{l}
\mathrm{Q}_{11 \mathrm{x}} \\
\mathrm{Q}_{11 \mathrm{y}} \\
\mathrm{Q}_{12 \mathrm{x}} \\
\mathrm{Q}_{12 \mathrm{y}} \\
\mathrm{Q}_{13 \mathrm{x}} \\
\mathrm{Q}_{13 \mathrm{y}} \\
\mathrm{Q}_{14 \mathrm{x}} \\
\mathrm{Q}_{14 \mathrm{y}}
\end{array}\right\}=\left\{\begin{array}{c}
0 \\
-8.125 \\
-3.51 \\
-8.125 \\
0 \\
-3.75 \\
-0.78 \\
-3.75
\end{array}\right\} \mathrm{kN}
$$

With the structural stiffness matrix and nodal forces, nodal displacements associated with each node can be calculated as,

$$
\{\mathrm{u}\}=\left\{\begin{array}{c}
\mathrm{u}_{5} \\
\mathrm{v}_{5} \\
\mathrm{u}_{6} \\
\mathrm{v}_{6} \\
\mathrm{u}_{7} \\
\mathrm{v}_{7} \\
\mathrm{u}_{8} \\
\mathrm{v}_{8} \\
\mathrm{u}_{9} \\
\mathrm{v}_{9} \\
\mathrm{u}_{10} \\
\mathrm{v}_{10} \\
\mathrm{u}_{11} \\
\mathrm{v}_{11} \\
\mathrm{u}_{12} \\
\mathrm{v}_{12} \\
\mathrm{u}_{13} \\
\mathrm{v}_{13} \\
\mathrm{u}_{14} \\
\mathrm{v}_{14}
\end{array}\right\}=10^{-3}\left\{\begin{array}{c}
-0.0002 \\
0.0007 \\
-0.0008 \\
-0.0051 \\
-0.0005 \\
0.001 \\
0.0007 \\
-0.003 \\
-0.066 \\
-0.019 \\
-0.066 \\
0.0013 \\
-0.016 \\
-0.024 \\
-0.163 \\
-0.0012 \\
-0.252 \\
-0.0257 \\
-0.251 \\
-0.002
\end{array}\right\} \mathrm{m}
$$

$$
\{\mathrm{F}\}=[\mathrm{K}]\{\mathrm{u}\}
$$

\section{B. Calculation of Stresses and Strains}

Strain induced in each element can be computed by

$$
\{\varepsilon\}=[\mathrm{B}]\{\mathrm{u}\}
$$

For Element 1, 
$\left\{\begin{array}{c}\epsilon_{\mathrm{x}} \\ \epsilon_{\mathrm{y}} \\ \gamma_{\mathrm{xy}}\end{array}\right\}=\left\{\begin{array}{c}-5^{*} 10^{-7} \eta-5^{*} 10^{-7} \\ -5.8 * 10^{-6} \xi-4.4 * 10^{-6} \\ -6^{*} 10^{-7} \xi-4.833 * 10^{-6} \eta-5.833 * 10^{-6}\end{array}\right\}$

Stresses induced in each element can be computed by

$\{\sigma\}=[C]\{\epsilon\}$

$\left\{\begin{array}{c}\sigma_{\mathrm{xx}} \\ \sigma_{\mathrm{yy}} \\ \tau_{\mathrm{xy}}\end{array}\right\}=\left\{\begin{array}{c}-40.28 \xi-15.93 \eta-55.78 \\ -161.11 \xi-5.472 \eta+0.076 \\ -6.25 \xi-50.34 \eta-50.98\end{array}\right\} \mathrm{kN} / \mathrm{m}^{2}$

In a similar way, strain and stress induced in other elements can also be computed.

For element 2,

$\left\{\begin{array}{c}\epsilon_{\mathrm{x}} \\ \epsilon_{\mathrm{y}} \\ \gamma_{\mathrm{xy}}\end{array}\right\}=\left\{\begin{array}{c}3.75^{*} 10^{-7} \eta+3.75^{*} 10^{-7} \\ 1.5 * 10^{-5} \xi+4.9^{*} 10^{-6} \\ 3 * 10^{-7} \xi+1.888 * 10^{-5} \eta+1.758^{*} 10^{-5}\end{array}\right\}$
$\left\{\begin{array}{c}\sigma_{\mathrm{xx}} \\ \sigma_{\mathrm{yy}} \\ \tau_{\mathrm{xy}}\end{array}\right\}=\left\{\begin{array}{c}104.86 \xi+10.417 \eta+44.444 \\ 419.44 \xi-22.77 \eta+138.71 \\ 3.125 \xi+196.61 \eta+183.07\end{array}\right\} \mathrm{kN} / \mathrm{m}^{2}$

For element 3,

$\left\{\begin{array}{c}\epsilon_{\mathrm{x}} \\ \epsilon_{\mathrm{y}} \\ \gamma_{\mathrm{xy}}\end{array}\right\}=\left\{\begin{array}{c}4 * 10^{-7} \xi+4 * 10^{-7} \\ 4 * 10^{-6} \xi-2 * 10^{-6} \\ -1.2 * 10^{-6} \xi-1.33^{*} 10^{-6} \eta-1.33 * 10^{-6}\end{array}\right\}$

$\left\{\begin{array}{l}\sigma_{\mathrm{xx}} \\ \sigma_{\mathrm{yy}} \\ \tau_{\mathrm{xy}}\end{array}\right\}=\left\{\begin{array}{c}11.108 \eta-27.778 \xi-2.78 \\ 21.27 \eta+111.13 \xi-127.32 \\ 12.5 \xi-13.885 \eta-11.802\end{array}\right\} \mathrm{kN} / \mathrm{m}^{2}$

For element 4,

$\left\{\begin{array}{c}\epsilon_{\mathrm{x}} \\ \epsilon_{\mathrm{y}} \\ \gamma_{\mathrm{xy}}\end{array}\right\}=\left\{\begin{array}{c}3.75^{*} 10^{-7}-3.75^{*} 10^{-7} \eta \\ 4.058^{*} 10^{-6} \xi-3.886^{*} 10^{-6} \\ 1.775 * 10^{-5} \eta-8.75^{*} 10^{-8} \xi-4.34 * 10^{-6}\end{array}\right\}$

$\left\{\begin{array}{l}\sigma_{\mathrm{xx}} \\ \sigma_{\mathrm{yy}} \\ \tau_{\mathrm{xy}}\end{array}\right\}=\left\{\begin{array}{c}28.178 \xi-10.417 \eta-16.571 \\ 112.71 \xi-2.604 \eta-105.35 \\ 184.89 \eta-0.893 \xi-45.286\end{array}\right\} \mathrm{kN} / \mathrm{m}^{2}$

For element 5,

$\left\{\begin{array}{c}\epsilon_{\mathrm{x}} \\ \epsilon_{\mathrm{y}} \\ \gamma_{\mathrm{xy}}\end{array}\right\}=\left\{\begin{array}{c}0 \\ 7.14 * 10^{-7} \xi-2.14 * 10^{-6} \\ 3.125^{*} 10^{-5} \eta-1.56^{*} 10^{-6}\end{array}\right\}$

$\left\{\begin{array}{l}\sigma_{\mathrm{xx}} \\ \sigma_{\mathrm{yy}} \\ \tau_{\mathrm{xy}}\end{array}\right\}=\left\{\begin{array}{l}4.961 \xi-14.883 \\ 19.844 \xi-59.53 \\ 32.55 \mathrm{\eta}-16.255\end{array}\right\} \mathrm{kN} / \mathrm{m}^{2}$

For element 6,

$\left\{\begin{array}{c}\epsilon_{\mathrm{x}} \\ \epsilon_{\mathrm{y}} \\ \gamma_{\mathrm{xy}}\end{array}\right\}=\left\{\begin{array}{c}1.25^{*} 10^{-6} \eta+1.25^{*} 10^{-6} \\ 2.992 * 10^{-7} \xi-8.331 * 10^{-7} \\ 3.333 * 10^{-7} \xi+1.125^{-7} 10^{-6} \eta-8.683 * 10^{-7}\end{array}\right\}$

$\left\{\begin{array}{l}\sigma_{\mathrm{xx}} \\ \sigma_{\mathrm{yy}} \\ \tau_{\mathrm{xy}}\end{array}\right\}=\left\{\begin{array}{c}2.083 \xi+34.722 \eta+28.936 \\ 8.33 \xi+8.681 \eta-14.462 \\ 3.471 \xi+11.719 \eta-8.691\end{array}\right\} \mathrm{kN} / \mathrm{m}^{2}$ 
If the value of normal and shear stress at any point is needed, it can be obtained by substituting the values of $\xi$ and $\eta$ in $\sigma_{\mathrm{xx}}, \sigma_{\mathrm{yy}}$ and $\tau_{\mathrm{xy}}$.

$\sigma_{\text {yy }}$ in the element $1=166.658 \mathrm{kN} / \mathrm{m}^{2}$

$\sigma_{\mathrm{yy}}$ in the element $3=5.08 \mathrm{kN} / \mathrm{m}^{2}$

\section{Calculation of stresses by static method [2,5]}

A cantilever retaining wall with uniform stem thickness and subjected to horizontal backfill for the chosen dimensions and properties is analyzed statically as shown in Table I.

From equilibrium,

Sum of Vertical forces, $\mathrm{R}_{\mathrm{v}}=216.25 \mathrm{kN}$

Sum of horizontal forces, $\mathrm{R}_{\mathrm{H}}=70.785 \mathrm{kN}$

Taking moment about toe,

$\mathrm{x}=(\Sigma$ Resisting moment $-\Sigma$ overturning moment $) / \mathrm{R}_{\mathrm{v}}$

$=(315.313-129.773) / 216.25=0.858 \mathrm{~m}$.

The resulting force lies within the middle third of the base.

Eccentricity, $\mathrm{e}=(\mathrm{B} / 2)-\mathrm{x}=1.25-0.858=0.392 \mathrm{~m}$

The Linear pressure distribution at the base is assumed .The maximum and minimum pressure is given by,

$\mathrm{p}_{\max }=\left(\mathrm{R}_{\mathrm{v}} / \mathrm{B}\right) *(1+(6 * \mathrm{e} / \mathrm{B}))=\mathbf{1 6 7 . 8 7 9} \mathrm{kN} / \mathrm{m}^{2}$

$\mathrm{p}_{\min }=\left(\mathrm{R}_{\mathrm{v}} / \mathrm{b}\right) *(1-(6 * \mathrm{e} / \mathrm{b}))=\mathbf{5 . 1 2 1} \mathrm{kN} / \mathrm{m}^{2}$

\section{TABLE I STATIC ANALYSIS OF CANTILEVER RETAINING WALL WITH UNIFORM STEM THICKNESS} AND SUBJECTED TO HORIZONTAL BACKFILL

\begin{tabular}{|c|c|c|c|c|}
\hline $\begin{array}{l}\text { S. } \\
\text { No. }\end{array}$ & $\begin{array}{l}\text { Vertical } \\
\text { Force }(\mathbf{k N})\end{array}$ & $\begin{array}{l}\text { Horizontal } \\
\text { Force }(k N)\end{array}$ & $\begin{array}{c}\text { Clockwise } \\
\text { Moment } \\
(\text { kNm) }\end{array}$ & $\begin{array}{l}\text { Anticlockwise } \\
\text { Moment (kNm) }\end{array}$ \\
\hline 1 & $25 * 0.4 * 5=50$ & - & $50 * 0.8=40$ & - \\
\hline 2 & $\begin{array}{c}25 * 2.5 * 0.5= \\
31.25\end{array}$ & - & $\begin{array}{c}31.25 * 1.25= \\
39.063\end{array}$ & - \\
\hline 3 & $\begin{array}{c}18 * 1.5 * 5= \\
135\end{array}$ & - & $\begin{array}{c}135 * 1.75= \\
236.25\end{array}$ & - \\
\hline 4 & - & $\begin{array}{l}0.5 * 25.74 * \\
5.5=70.785\end{array}$ & - & $\begin{array}{c}70.785 * 5.5 / 3= \\
129.773\end{array}$ \\
\hline$\Sigma$ & 216.25 & 70.785 & 315.313 & 129.773 \\
\hline
\end{tabular}

\section{Check for Overturning}

The factor of safety for overturning of the cantilever retaining wall is calculated by taking moments about the toe of the foundation.

Factor of safety $=\frac{\text { Sum of resisting moment about toe }}{\text { Sum of overturning moment about toe }}$

$=\frac{315.313}{129.773}=2.43>2$. 
The minimum factor of safety for overturning is 1.5 to 2.0 . Hence the cantilever retaining wall considered is safe against overturning.

\section{Check for Sliding}

The factor of safety for sliding of the cantilever retaining wall is often obtained as the resisting force divided by the driving force.

Factor of Safety $=\frac{\angle \mathrm{R}_{\mathrm{v}}}{\mathrm{R}_{\mathrm{H}}}=\frac{0.577 * 216.25}{70.785}=1.763$

$\angle=\tan \mathrm{d}$

where,

$\angle$-Coefficient of friction between the base of the wall and the soil

$\mathrm{d}$ - Friction angle between the base of the wall and the foundation

The minimum factor of safety for sliding is 1.5 to 2.0 . Hence the cantilever retaining wall considered is safe against sliding.

$\angle=\tan \mathrm{d}$

where,

$\angle$-Coefficient of friction between the base of the wall and the soil

$\mathrm{d}$ - Friction angle between the base of the wall and the foundation

The minimum factor of safety for sliding is 1.5 to 2.0 . Hence the cantilever retaining wall considered is safe against sliding.

\section{Analysis Of Cantilever Retaining Wall Using Ansys}

A Cantilever retaining wall considered in this study is modeled and analyzed using ANSYS.

\section{A. Modeling of Cantilever Retaining wall}

A Cantilever retaining wall for the chosen dimensions and properties is modeled as a 2D plane strain model as shown in Fig.7.

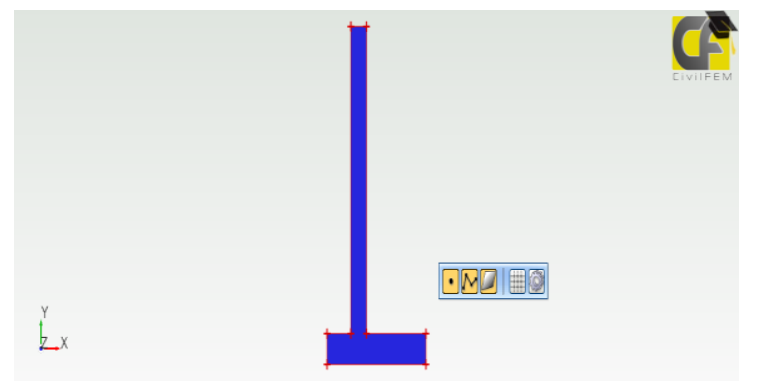

Fig. 7 Cantilever Retaining Wall with Uniform Stem Thickness model

In order to mesh the Cantilever retaining wall, it is necessary to obtain the optimum edge size of an element. It can be obtained by determining the convergence of the ycomponent stress $\left(\sigma_{\mathrm{yy}}\right)$ as shown in Table II 
TABLE II STRESS $\left(\sigma_{\mathrm{YY}}\right)$ AT TOE FOR CORRESPONDING EDGE SIZE

\begin{tabular}{|c|c|c|}
\hline S.No. & Edge Size $(\mathbf{m})$ & $\begin{array}{c}\text { Stress }\left(\boldsymbol{\sigma}_{\mathbf{Y Y}}\right) \text { at Toe } \\
(\mathbf{k N} / \mathbf{m} \mathbf{2})\end{array}$ \\
\hline 1 & 0.05 & 168.225 \\
\hline 2 & 0.1 & 168.860 \\
\hline 3 & 0.15 & 169.007 \\
\hline 4 & 0.2 & 169.159 \\
\hline 5 & 0.25 & 168.973 \\
\hline 6 & 0.3 & 168.186 \\
\hline $\mathbf{7}$ & $\mathbf{0 . 3 5}$ & $\mathbf{1 6 8 . 2 6 1}$ \\
\hline 8 & 0.4 & 168.468 \\
\hline
\end{tabular}

Since the stress value converges at an edge size of $0.35 \mathrm{~m}$, the optimum edge size is 0.35 m.

\section{B. Meshing the Cantilever Retaining wall}

The Quadrangle type of element with an optimum edge size of $0.35 \mathrm{~m}$ is used for meshing the cantilever retaining wall. As a result of meshing, there are 70 nodes and 44 elements in the cantilever retaining wall as shown in Fig.8.

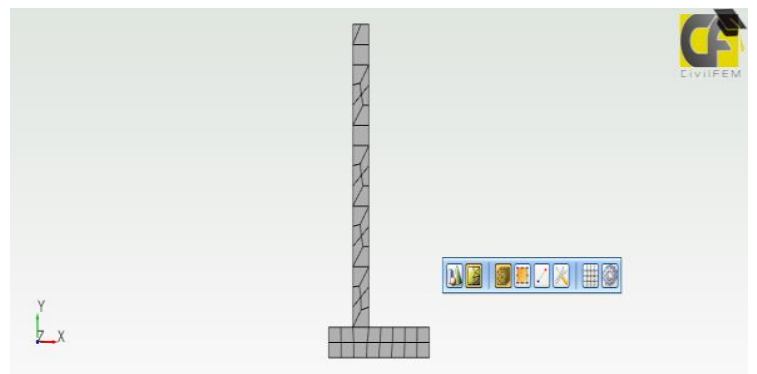

Fig. 8 Meshing of Cantilever Retaining Wall with Uniform Stem Thickness

Cantilever retaining wall which is fixed at the bottom is subjected to an active earth pressure of $25.74 \mathrm{kN} / \mathrm{m} 2$ at the bottom and zero at top in addition to its self weight as shown in Fig. 9

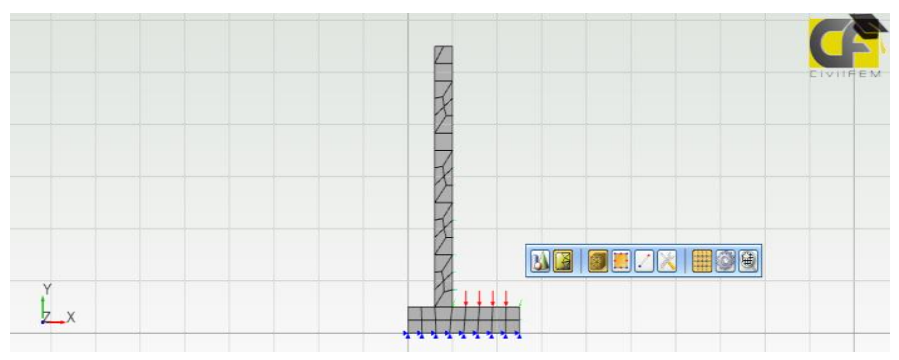

Fig. 9 Cantilever Retaining Wall with Uniform Stem Thickness Carrying Loads 


\section{Stress Distribution of Cantilever Retaining Wall with Uniform Stem Thickness}

After applying the loads with the proper boundary conditions, the stress distribution of the cantilever retaining wall obtained is shown in Fig. 10

Stress at toe $=\mathbf{1 6 8 . 2 6 1} \mathrm{kN} / \mathrm{m}^{2}$

Stress at heel $=\mathbf{5 . 1 3 5} \mathrm{kN} / \mathrm{m}^{2}$

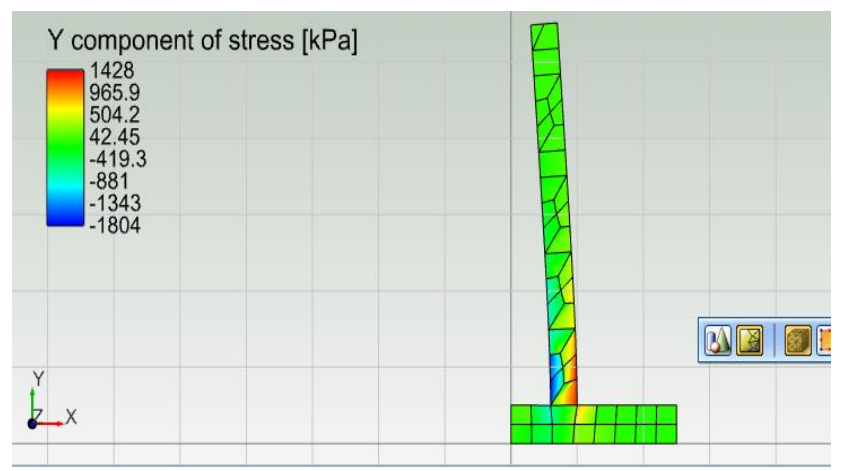

Fig. 10 Stress Pattern of Cantilever Retaining Wall with Uniform Stem Thickness

\section{Comparison Of Stresses Induced In The Cantilever Retaining Wall}

The stresses induced in Cantilever retaining wall from different methods are compared and given in Table III and Fig 11 respectively.

TABLEIII STRESSES INDUCED IN CANTILEVER RETAINING WALL OBTAINED FROM DIFFERENT METHODS

\begin{tabular}{|c|c|c|c|}
\hline $\begin{array}{c}\text { Stress, } \\
\sigma \mathbf{y y} \\
(\mathbf{k N} / \mathbf{m} 2)\end{array}$ & $\begin{array}{c}\text { Matrix } \\
\text { Displacement } \\
\text { method }\end{array}$ & ANSYS & $\begin{array}{c}\text { Static } \\
\text { method }\end{array}$ \\
\hline At toe & 152.154 & 153.103 & 154.864 \\
\hline At heel & 94.038 & 94.291 & 96.453 \\
\hline
\end{tabular}

\section{Conclusion}

In this paper, the efficiency of Matrix Displacement method on the solution of plane strain problem is investigated by considering a cantilever retaining wall as an example. The Stress values of the cantilever retaining wall subjected to lateral loads are obtained using Matrix Displacement method and are compared with the results obtained from ANSYS and the Static method. The Stresses at toe and heel of the Cantilever retaining wall obtained by Matrix Displacement method varies from that obtained by ANSYS by $0.953 \%$ and $1.07 \%$ respectively. The Stresses at toe and heel of the Cantilever retaining wall obtained by Matrix Displacement method varies from that obtained by Static method by $0.727 \%$ and $.8 \%$ respectively.Based on the results 
obtained from this study, the Matrix Displacement method can be used effectively for the stress analysis of cantilever retaining walls. Further studies can be carried out to prove the efficiency of Matrix Displacement method on the solution of plane stress and plane strain problems using different types of structures.

\section{ACKNOWLEDGEMENT}

The authors wish to express their sincere gratitude to the Principal and the Head of the Department of Civil Engineering PSG College of Technology, Coimbatore for providing the necessary facilities to carry out the work reported in this paper.

\section{References}

[1] Anuj Chandiwala K, Gunvant solanki, Yash chaliawala (2015), "Comparative study on cantilever and counterfort retaining wall", International journal of Advance Engineering and Research Development, 2 (12), 221-224.

[2] Bharat Shah \& Tapkaire P P (2015), "Optimization of gravity retaining wall profile by introducing cavity", International Journal of Civil, Structural, Environmental and Infrastructure Engineering Research and Development, 5 (4), 87-96.

[3] Logan D L (2002), "A First Course in Finite Element Method", Thomson \& Brooks/Cole.

[4] Mustafa Ergun and Sevket Ates (2015), 'The stress analysis of shear wall with matrix displacement method", Structural Engineering and Mechanics, 53 (2), 205-226.1

[5] D. S. Vijayan and J. J. Daniel, "An investigation on the torsional effect of symmetric moment resisting frame system subjected to eccentric reinforced concrete lift wall - A finite element approach," Int. J. Eng. Trends Technol., vol. 69, no. 8, pp. 179-184, 2021, doi: 10.14445/22315381/IJETT-V69I8P222.

[6] Potts D M \& Zdrakovic L (2001), "Finite element Analysis in Geotechnical Engineering",Thomas Telford Books, London.

[7] D.S.Vijayan, S.Arvindan, K.Naveen Kumar, S Mohamed Javed, Seismic Performance of Flat Slab in Tall Buildings with and without a Shear wall, International Journal of Engineering and Advanced Technology (IJEAT) ISSN: 2249 - 8958, Volume-9 Issue-1, October 2019, Page no $-2672-2675$.

[8] Rajasekaran S (2003), "Finite Element Analysis in Engineering Design", S Chand \& Co.,

[9] Rao S S (2005),"The Finite Element Method in Engineering", Elsevier Science and Technology Books. 